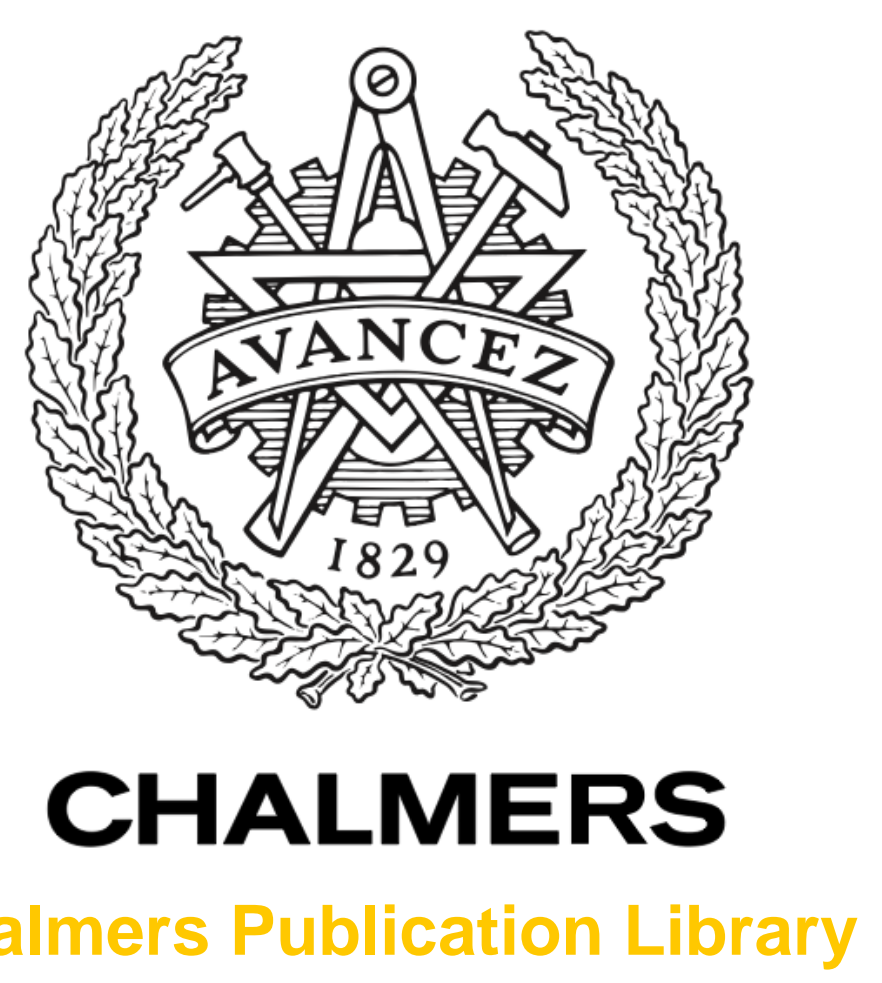

Chalmers Publication Library

\title{
A CPHD Filter for Tracking With Spawning Models
}

This document has been downloaded from Chalmers Publication Library (CPL). It is the author's version of a work that was accepted for publication in:

IEEE Journal on Selected Topics in Signal Processing (ISSN: 1932-4553)

Citation for the published paper:

Lundgren, M. ; Svensson, L. ; Hammarstrand, L. (2013) "A CPHD Filter for Tracking With Spawning Models". IEEE Journal on Selected Topics in Signal Processing, vol. 7(3), pp. 496-507.

http://dx.doi.org/10.1109/JSTSP.2013.2252599

Downloaded from: http://publications.lib.chalmers.se/publication/179584

Notice: Changes introduced as a result of publishing processes such as copy-editing and formatting may not be reflected in this document. For a definitive version of this work, please refer to the published source. Please note that access to the published version might require a subscription.

Chalmers Publication Library (CPL) offers the possibility of retrieving research publications produced at Chalmers University of Technology. It covers all types of publications: articles, dissertations, licentiate theses, masters theses, conference papers, reports etc. Since 2006 it is the official tool for Chalmers official publication statistics. To ensure that Chalmers research results are disseminated as widely as possible, an Open Access Policy has been adopted.

The CPL service is administrated and maintained by Chalmers Library. 


\title{
A CPHD Filter for Tracking with Spawning Models
}

\author{
Malin Lundgren, Lennart Svensson, Senior Member, IEEE, Lars Hammarstrand
}

\begin{abstract}
In some applications of multi-target tracking, appearing targets are suitably modeled as spawning from existing targets. However, in the original formulation of the cardinalized probability hypothesis density (CPHD) filter, this type of model is not supported; instead appearing targets are modeled by spontaneous birth only. In this paper we derive the necessary equations for a CPHD filter for the case when the process model also includes target spawning. For this generalized filter, the cardinality prediction formula might become computationally intractable for general spawning models. However, when the cardinality distribution of the spawning targets is either Bernoulli or Poisson, we derive expressions that are practical and computationally efficient. Simulations show that the proposed filter responds faster to a change in target number due to spawned targets than the original CPHD filter. In addition, the performance of the filter, considering the optimal subpattern assignment (OSPA), is improved when having an explicit spawning model.
\end{abstract}

Index Terms-Bayesian methods, Filtering theory, Recursive estimation

\section{INTRODUCTION}

Multi-target tracking is used to detect and position an unknown and varying number of objects using sensor observations affected by noise and clutter. The problem includes data association and detection uncertainties, originating from the fact that a target might not be detected and that the source of each measurement is unknown. Traditionally, as in [1], [2], tracking algorithms have been designed using target labeling which means that the algorithm discriminates between different targets. Examples of such algorithms are the Probabilistic Data Association (PDA) filter [3], the Joint PDA (JPDA) filter [4] and the Multiple Hypothesis Tracking (MHT) algorithm [5], [6]. During the last decade, however, a family of alternative methods that, in its original form, disregards target labels has gained a lot of research attention. The idea is that the collection of target states can be described by a random finite set (RFS) [7], a representation that captures the randomness in the number of targets and their states, while not discriminating between targets since the set is unordered. Recent results [8], however, show that target labels can naturally be incorporated into these methods albeit at the expense of increased computational complexity. Using RFSs to model the multi-target state and the received observations, the

Copyright (c) 2013 IEEE. Personal use of this material is permitted. However, permission to use this material for any other purposes must be obtained from the IEEE by sending a request to pubs-permissions@ieee.org This work was supported by the Strategic Vehicle Research and Innovation Program (FFI), which is funded by the Swedish Agency for Innovation Systems (VINNOVA). M. Lundgren, L. Svensson and L. Hammarstrand are with the Department of Signals and Systems, Chalmers University of Technology, SE-412 96, Gothenburg, Sweden (E-mail: \{malin.lundgren,lennart.svensson,lars.hammarstrand\}@ chalmers.se). tracking problem can be formulated in a Bayesian framework in a theoretically solid fashion.

Similar to the traditional case, where the full Bayesian filter is rarely used, the set-based Bayes filter has, until recently [8], been considered intractable in practise. To have a more practical method, the probability hypothesis density (PHD) filter [9] was developed. This filter finds an approximate solution by, at each time instance, only considering the first order moment of the multi-target density, called the intensity function. The intensity function describes the number of targets and their distribution on the single target state space, and in the PHD filter the intensity is approximated in a way implying that the number of targets is Poisson distributed. As a consequence, the variance is equal to the expected number of targets, which may lead to problems in the estimation of the number of targets [10]. To address this, the cardinalized probability hypothesis density (CPHD) filter was derived in [11]. In addition to the intensity function, the CPHD filter also propagates the full probability mass function for the number of targets, called the cardinality distribution. In contrast to the PHD filter, the cardinality distribution in CPHD is arbitrary.

In order to have a practical implementation of the PHD and the CPHD filters it is necessary to have a tractable representation of the intensity function. Two alternatives are the sequential Monte Carlo/particle filter versions [12] and the Gaussian mixture versions [13], [14] of the filters, where the latter is the most commonly used. Moreover, a method for deriving the PHD and CPHD recursions, without using finite set statistics (FISST), is presented in [15], [16].

Since they were introduced, PHD and CPHD filters have been applied to a variety of problems and have been modified to handle different models and modeling assumptions. Examples of such filter versions are the CPHD filter for unknown clutter intensity and unknown detection probability [17], the PHD/CPHD filter for tracking extended targets, i.e., where more than one measurement can originate from the same target [18]-[20] and the PHD filter for jump Markov models [21]. However, to the best of our knowledge, there has thus far not been a CPHD filter including a model for spawning targets. Within the area of target tracking, spawning means that appearing targets can be modeled as generated by existing targets. Examples of such situations can be when airplanes take off from a carrier boat or when a sensor resolves new features on an extended object. Target spawning is incorporated into the original PHD filter but not into the CPHD filter where new targets are modeled by spontaneous birth only.

In this paper we generalize the CPHD filter to consider target spawning, by incorporating an explicit model describing the appearance of these targets. In contrast to the original CPHD filter, the derivations are here performed using traditional Bayesian statistics and therefore no background within 
finite set statistics (FISST) is needed. For a general spawning model, the resulting prediction equation for the cardinality distribution turns out to be impractical. However, we show that it is possible to find tractable expressions when the number of spawned targets from a single target follows either a Bernoulli or a Poisson distribution. Evaluation, comparing the generalized CPHD filter to the original filter, is performed on a scenario where new targets appear through spawning from existing targets. The results show that we gain in performance, considering the optimal subpattern assignment (OSPA) measure [22], [23], by adding a spawning model compared to the original CPHD filter.

The paper is organized as follows. Section II describes the multi-target tracking problem and gives the prerequisites for an RFS based solution. Some RFS theory and an overview of the PHD and CPHD filters in given in Section III. More precisely, the section summarizes the assumptions and approximations that the two filters are based upon and state the filter equations required for one recursion. Based on this, in Section IV, the necessary equations for a CPHD filter incorporating an explicit spawning model is derived. Section V considers specific spawning and birth models, and how they affect the final filter equations. Simulation results are presented in Section VI and, finally, Section VII concludes the paper.

\section{PROBLEM FORMULATION}

In this paper we consider the problem of tracking an unknown and varying number of targets using sensor measurements that are affected by noise and clutter. At the discrete time instance $k$, we denote the number of targets by $N_{k}$, and their individual states by $\mathbf{x}_{k}^{1}, \mathbf{x}_{k}^{2}, \ldots, \mathbf{x}_{k}^{N_{k}}$, which take values in the single-target state space $\mathcal{X} \subseteq \mathbb{R}^{n_{x}}$. The state vector of a target can, for example, describe position, velocity and acceleration of the target. The complete multi-target state at time $k$ is defined as the unordered set of the single target states,

$$
X_{k}=\left\{\mathbf{x}_{k}^{1}, \mathbf{x}_{k}^{2}, \ldots, \mathbf{x}_{k}^{N_{k}}\right\} .
$$

Since the number of targets as well as the target states are random variables, $X_{k}$ is a random finite set (RFS) [7].

An RFS is described by a probability mass function of its cardinality, and a family of joint probability densities of the target states. In the multi-target tracking problem considered in this paper, the aim is to estimate both the cardinality and the individual target states, given all measurements up to the current time instant. To evaluate the performance of the tracking filter we use the OSPA metric [22], which takes into account both the cardinality and the state estimation, but does not consider target labels. Thus, it fits well with the set representation of the targets.

\section{A. Process model}

Going from the discrete time instance $k-1$ to $k$, a target with state $\mathbf{x}_{k-1}$ can either continue to exist or disappear, with probabilities $P_{\mathrm{s}}\left(\mathbf{x}_{k-1}\right)$ and $1-P_{\mathrm{s}}\left(\mathbf{x}_{k-1}\right)$, respectively. Conditioned on the existence of a target $\mathbf{x}_{k-1}$ at time $k-1$, the target evolves independently of all other targets according to a transition density $p\left(\mathbf{x}_{k} \mid \mathbf{x}_{k-1}\right)$. Using the RFS notation, the evolution of a target can be described by the RFS $S\left(\mathbf{x}_{k-1}\right)$, which is $\{\emptyset\}$ with probability $1-P_{\mathrm{s}}\left(\mathbf{x}_{k-1}\right)$ and a singleton $\left\{\mathbf{u}_{x}\left(\mathbf{x}_{k-1}\right)\right\}$ with probability $P_{\mathrm{s}}\left(\mathbf{x}_{k-1}\right)$, where $\mathbf{u}_{x}\left(\mathbf{x}_{k-1}\right)$ is a random vector whose distribution is $p\left(\mathbf{x}_{k} \mid \mathbf{x}_{k-1}\right)$.

In addition to existing targets, at each time instant new targets might appear at the scene, which can happen either through spontaneous birth or through spawning from existing targets. These new targets are described by two independent RFSs, the birth RFS $\Gamma_{k}$ and the spawn RFS $B(\mathbf{x})$. The complete multi-target state at time $k$ is written as the union

$$
X_{k}=\left[\bigcup_{\mathbf{x} \in X_{k-1}} S(\mathbf{x})\right] \cup\left[\bigcup_{\mathbf{x} \in X_{k-1}} B(\mathbf{x})\right] \cup \Gamma_{k} .
$$

This equation thus describes how the target RFS, i.e both the number of targets and their states, changes over time and the aim in this paper is to incorporate this model into the wellknown CPHD filter.

\section{B. Measurement model}

At each time $k$, a set of $m_{k}$ measurements is received. The number of received measurements varies with time and can be collected in the set

$$
Z_{k}=\left\{\mathbf{z}_{k}^{1}, \mathbf{z}_{k}^{2}, \ldots, \mathbf{z}_{k}^{m_{k}}\right\}
$$

where each measurement $\mathbf{z}_{k}^{i}$ takes on values in the measurement space $\mathcal{Z} \subseteq \mathbb{R}^{n_{z}}$. This set consists of both target generated measurements and clutter, and it is unknown which measurements originate from the targets and which are clutter.

Each target $\mathbf{x}_{k}$ is either detected or not, independently of all other targets, with probabilities $P_{\mathrm{d}}\left(\mathbf{x}_{k}\right)$ and $1-P_{\mathrm{d}}\left(\mathbf{x}_{k}\right)$, respectively. Conditional on the detection of a target with state $\mathbf{x}_{k}$, the produced measurement is described by the density $p\left(\mathbf{z}_{k} \mid \mathbf{x}_{k}\right)$. Similarly as for the survival process, the detection of a target can be described by the RFS $T\left(\mathbf{x}_{k}\right)$ which is $\{\emptyset\}$ with probability $1-P_{\mathrm{d}}\left(\mathbf{x}_{k}\right)$ and $\left\{\mathbf{u}_{z}\left(\mathbf{x}_{k}\right)\right\}$ with probability $P_{\mathrm{d}}\left(\mathbf{x}_{k}\right)$, where $\mathbf{u}_{z}\left(\mathbf{x}_{k}\right) \sim p\left(\mathbf{z}_{k} \mid \mathbf{x}_{k}\right)$.

The received set of measurements can be written as the union of target-generated measurements and the clutter RFS $K_{k}$,

$$
Z_{k}=\left[\bigcup_{\mathbf{x} \in X_{k}} T(\mathbf{x})\right] \cup K_{k} .
$$

Finally, the collection of all measurements up to time $k$ is denoted $Z_{1: k}$, and conditioned on this set we aim to find an estimate of the target RFS, $X_{k}$, at time $k$.

\section{BACKGROUND OF PHD AND CPHD}

In this section we describe how to deal with random finite sets in a Bayesian filtering framework, a problem that has gained a lot of research attention during recent years. The discussion starts with the multi-target Bayes filter and continues with the two most common finite set statistics (FISST) filters in practise, namely the probability hypothesis density (PHD) filter [9] and the cardinalized probability hypothesis density (CPHD) filter [11]. 
When dealing with multi-target tracking, while representing targets and measurements as sets, we need an alternative Bayesian filter that is able to handle set densities. In [7], the multi-target Bayesian filter is given by the equations

$$
\begin{aligned}
p\left(X_{k} \mid Z_{1: k-1}\right) & =\int p\left(X_{k} \mid X_{k-1}\right) p\left(X_{k-1} \mid Z_{1: k-1}\right) \delta X_{k-1} \\
p\left(X_{k} \mid Z_{1: k}\right) & =\frac{p\left(Z_{k} \mid X_{k}\right) p\left(X_{k} \mid Z_{1: k-1}\right)}{\int p\left(Z_{k} \mid X_{k}\right) p\left(X_{k} \mid Z_{1: k-1}\right) \delta X_{k}},
\end{aligned}
$$

where the integrals are set integrals defined as,

$$
\int p(X) \delta X=p(\emptyset)+\sum_{n=1}^{\infty} \frac{1}{n !} \int p\left(\left\{\mathbf{x}_{1}, \ldots, \mathbf{x}_{n}\right\}\right) d \mathbf{x}_{1} \cdots d \mathbf{x}_{n}
$$

This integral considers both the variability in the number of elements in the set $X$, as well as the distribution of the elements.

Until recently, the full Bayesian filter has been considered computationally intractable, which has led to the development of approximate solutions, like the PHD and the CPHD filters. Instead of propagating the full multi-target density, the PHD filter propagates its first order moment, called the intensity function, while the CPHD filter propagates both the intensity function and the cardinality distribution of the RFS. The intensity function, $v(\mathbf{x})$, of an RFS $X$ is defined by the property

$$
\int_{S} v(\mathbf{x}) d \mathbf{x}=E\{|X \cap S|\}
$$

where $S$ is a region in the single-target state space, and $|X|$ is the notation for the cardinality of the set $X$. Hence, the integral in (8) gives the expected number of targets in $X$ that are in the region $S$, and similarly $\int v(\mathbf{x}) d \mathbf{x}$ gives the expected number of targets in $X$. The cardinality distribution of an RFS is the probability mass function $p(n)$, which gives the probability that the set contains exactly $n$ elements/targets.

The remainder of this section briefly summarizes the PHD filter and the CPHD filter. We give some background, state the assumptions on which the filters are based, and present the equations needed for one filter recursion. This is later on used as a base for further discussions and the derivations of the CPHD filter with spawning.

Notation 1: In this paper the notation $\langle\alpha, \beta\rangle$ is used for the inner product between $\alpha$ and $\beta$, i.e.

$\langle\alpha, \beta\rangle= \begin{cases}\int \alpha(x) \beta(x) d x & \text { if } \alpha \text { and } \beta \text { are continuous function } \\ \sum \alpha(m) \beta(m) & \text { if } \alpha \text { and } \beta \text { are discrete functions. }\end{cases}$

Notation 2: For probability densities and intensity functions we often incorporate the time indices according to

$$
\begin{aligned}
p_{k \mid k-1}(\mathbf{x}) & =p\left(\mathbf{x}_{k}=\mathbf{x} \mid Z_{1: k-1}\right) \\
p_{k \mid k-1}\left(\mathbf{x} \mid \mathbf{x}^{\prime}\right) & =p\left(\mathbf{x}_{k}=\mathbf{x} \mid \mathbf{x}_{k-1}=\mathbf{x}^{\prime}\right) .
\end{aligned}
$$

\section{A. The probability hypothesis density filter}

The PHD filter only propagates the intensity function over time, and each iteration in the filter consists of a prediction and a measurement update of the intensity function. The derivation of the filter equations is based on the following assumptions and approximations:

- The predicted multi-target RFS is a Poisson process, i.e. the targets are assumed to be independent and identically distributed (i.i.d.) with a Poisson cardinality distribution.

- Each target evolves and generates measurements independently of all other targets. The evolution of a target is described by the single-target Markov density $p_{k \mid k-1}\left(\mathbf{x} \mid \mathbf{x}^{\prime}\right)$, and a target-generated measurement by the likelihood $L_{k, \mathbf{z}}(\mathbf{x})=p\left(\mathbf{z}_{k}=\mathbf{z} \mid \mathbf{x}_{k}=\mathbf{x}\right)$.

- The birth of new targets and the survival of existing targets are independent of each other.

- New targets can appear through spontaneous birth or through spawning from existing targets. The intensity function of the birth process is denoted $b_{k}(\mathbf{x})$ and the spawning intensity for a single target with state $\mathbf{x}^{\prime}$ is given by $s_{k \mid k-1}\left(\mathbf{x} \mid \mathbf{x}^{\prime}\right)$.

- The clutter RFS is a Poisson process with cardinality distribution $p_{\mathrm{c}}(n)=e^{-\lambda} \lambda^{n} / n$ !, probability density $c(\mathbf{z})$ and intensity function $\lambda c(\mathbf{z})$.

- The clutter measurements are independent of the targetgenerated measurements.

Under these assumptions, and using finite set statistics, the PHD filter prediction and measurement update equations were derived in [9]. The equations are summarized here:

Prediction: Suppose that the intensity function, $v_{k-1 \mid k-1}(\mathbf{x})$, is given from time $k-1$. Then the predicted intensity is given by

$$
\begin{aligned}
v_{k \mid k-1}(\mathbf{x})= & b_{k}(\mathbf{x})+\int\left\{P_{\mathrm{s}}\left(\mathbf{x}^{\prime}\right) p_{k \mid k-1}\left(\mathbf{x} \mid \mathbf{x}^{\prime}\right) v_{k-1 \mid k-1}\left(\mathbf{x}^{\prime}\right)\right. \\
& \left.+s_{k \mid k-1}\left(\mathbf{x} \mid \mathbf{x}^{\prime}\right) v_{k-1 \mid k-1}\left(\mathbf{x}^{\prime}\right)\right\} d \mathbf{x}^{\prime}
\end{aligned}
$$

Update: Suppose that the predicted intensity function, $v_{k \mid k-1}(\mathbf{x})$, is given at time $k$, together with the set of measurements, $Z_{k}=\left\{\mathbf{z}_{1}, \ldots, \mathbf{z}_{m_{k}}\right\}$. Then, the intensity function after measurement update is given by

$$
\begin{aligned}
v_{k \mid k}(\mathbf{x})= & {\left[\sum_{\mathbf{z} \in Z_{k}} \frac{P_{\mathrm{d}}(\mathbf{x}) L_{k, \mathbf{z}}(\mathbf{x})}{\lambda c(\mathbf{z})+\left\langle v_{k \mid k-1}, P_{\mathrm{d}} L_{k, \mathbf{z}}\right\rangle}\right.} \\
& \left.+\left(1-P_{\mathrm{d}}(\mathbf{x})\right)\right] \cdot v_{k \mid k-1}(\mathbf{x}) .
\end{aligned}
$$

\section{B. The cardinalized probability hypothesis density filter}

The CPHD filter propagates both the intensity function, $v(\mathbf{x})$, and the cardinality distribution, $p(n)$, over time. Each iteration consists of a prediction and a measurement update of both the intensity and the cardinality. The assumptions on which the filter is based are similar to that of the PHD filter, but differs at some points. Two important differences are that the cardinality distribution of the target RFS is approximated as Poisson in PHD, while it is arbitrary in CPHD, and while new targets might appear through spontaneous birth and spawning 
in PHD, they can only appear through birth in the CPHD filter. Hence, the derivations are based on the following assumptions:

- The prior and the predicted RFSs are i.i.d. cluster processes, i.e. the targets are assumed to be independent and identically distributed with an arbitrary cardinality distribution.

- Each target evolves and generates measurements independently of all other targets. The evolution of a target is described by the single-target Markov density $p_{k \mid k-1}\left(\mathbf{x} \mid \mathbf{x}^{\prime}\right)$, and a target-generated measurement by the likelihood $L_{k, \mathbf{z}}(\mathbf{x})=p\left(\mathbf{z}_{k}=\mathbf{z} \mid \mathbf{x}_{k}=\mathbf{x}\right)$.

- The birth and survival of targets are independent of one another.

- New targets appear through spontaneous birth described by the intensity $b_{k}(\mathbf{x})$ and cardinality distribution $p_{\mathrm{b}}(n)$.

- The clutter RFS is an i.i.d. cluster process with cardinality distribution $p_{\mathrm{c}}(n)$, probability density $c(\mathbf{z})$ and intensity function $\kappa(\mathbf{z})$.

- The clutter measurements are independent of the targetgenerated measurements.

Under these assumptions, the prediction and the measurement update equations were derived in [11]. The update equations presented here are not from the original recursion, but instead the equivalent ones stated in [24], in order to have expressions that do not involve probability generating functions.

Prediction: Suppose that the intensity function, $v_{k-1 \mid k-1}(\mathbf{x})$, and the cardinality distribution, $p_{k-1 \mid k-1}(n)$, are given from time $k-1$. Then the predicted intensity and the predicted cardinality distribution are given by

$$
v_{k \mid k-1}(\mathbf{x})=b_{k}(\mathbf{x})+\int P_{\mathrm{s}}\left(\mathbf{x}^{\prime}\right) p_{k \mid k-1}\left(\mathbf{x} \mid \mathbf{x}^{\prime}\right) v_{k-1 \mid k-1}\left(\mathbf{x}^{\prime}\right) d \mathbf{x}^{\prime}
$$

$$
\begin{aligned}
p_{k \mid k-1}(n) & =\sum_{j=0}^{n} p_{\mathrm{b}}(n-j) \sum_{m=j}^{\infty}\left(\begin{array}{c}
m \\
j
\end{array}\right)\left\langle P_{\mathrm{s}}, v_{k-1 \mid k-1}\right\rangle^{j} \\
& \times \frac{\left\langle\left(1-P_{\mathrm{s}}\right), v_{k-1 \mid k-1}\right\rangle^{m-j}}{\left\langle 1, v_{k-1 \mid k-1}\right\rangle^{m}} p_{k-1 \mid k-1}(m)
\end{aligned}
$$

Update: Suppose that the predicted intensity function, $v_{k \mid k-1}(\mathbf{x})$, and the predicted cardinality distribution, $p_{k \mid k-1}(n)$, are given at time $k$. A set of measurement, $Z_{k}=\left\{\mathbf{z}_{1}, \ldots, \mathbf{z}_{m_{k}}\right\}$, is also given. Then, the intensity function and cardinality distribution after measurement update are given by

$$
\begin{aligned}
v_{k \mid k}(\mathbf{x}) & =\left[\sum_{\mathbf{z} \in Z_{k}} \frac{\left\langle\Upsilon_{k}^{1}\left[v_{k \mid k-1}, Z_{k} \backslash\{\mathbf{z}\}\right], p_{k \mid k-1}\right\rangle}{\left\langle\Upsilon_{k}^{0}\left[v_{k \mid k-1}, Z_{k}\right], p_{k \mid k-1}\right\rangle} \cdot \psi_{k, \mathbf{z}}(\mathbf{x})\right. \\
& \left.+\frac{\left\langle\Upsilon_{k}^{1}\left[v_{k \mid k-1}, Z_{k}\right], p_{k \mid k-1}\right\rangle}{\left\langle\Upsilon_{k}^{0}\left[v_{k \mid k-1}, Z_{k}\right], p_{k \mid k-1}\right\rangle}\left(1-P_{\mathrm{d}}(\mathbf{x})\right)\right] v_{k \mid k-1}(\mathbf{x}) \\
p_{k \mid k}(n) & =\frac{\Upsilon_{k}^{0}\left[v_{k \mid k-1}, Z_{k}\right](n) \cdot p_{k \mid k-1}(n)}{\left\langle\Upsilon_{k}^{0}\left[v_{k \mid k-1}, Z_{k}\right], p_{k \mid k-1}\right\rangle}
\end{aligned}
$$

where

$$
\begin{aligned}
\Upsilon_{k}^{u}[v, Z] & =\sum_{j=0}^{\min (|Z|, n)}(|Z|-j) ! p_{\mathrm{c}}(|Z|-j) \frac{n !}{(n-j) !} \\
& \times \frac{\left\langle 1-P_{\mathrm{d}}, v\right\rangle^{n-(j+u)}}{\langle 1, v\rangle^{n}} \sigma_{j}(\Phi(v, Z)) \\
\Phi(v, Z) & =\left\{\left\langle v, \psi_{k, \mathbf{z}}\right\rangle: \mathbf{z} \in Z\right\} \\
\psi_{k, \mathbf{z}}(\mathbf{x}) & =\frac{\langle 1, \kappa\rangle}{\kappa(\mathbf{z})} L_{k, \mathbf{z}}(\mathbf{x}) P_{\mathrm{d}}(\mathbf{x})=\frac{L_{k, \mathbf{z}}(\mathbf{x}) P_{\mathrm{d}}(\mathbf{x})}{c(\mathbf{z})},
\end{aligned}
$$

and where the function $\sigma_{j}(\mathcal{S})$ is called the elementary symmetric function of order $j$, which is defined as the sum over all possible products of $j$ elements from the set $\mathcal{S}$

$$
\sigma_{j}\left(\left\{y_{1}, y_{2}, \ldots, y_{n}\right\}\right)=\sum_{1 \leq i_{1}<i_{2}<\ldots<i_{j} \leq n} y_{i_{1}} \cdot y_{i_{2}} \cdots y_{i_{j}} .
$$

\section{Gaussian mixture implementation}

In order to have a practical implementation of the PHD and the CPHD filters, we need a representation of the intensity function that is easy to handle. In the Gaussian mixture versions of PHD and CPHD it is assumed that the intensity function can be described as a weighted sum of Gaussians

$$
v_{k \mid k}(\mathbf{x})=\sum_{j=0}^{J_{k \mid k}} w_{k \mid k}^{(j)} \mathcal{N}\left(\mathbf{x} ; \mathbf{m}_{k \mid k}^{(j)}, \mathbf{P}_{k \mid k}^{(j)}\right)
$$

where $\mathbf{m}_{k \mid k}^{(j)}$ and $\mathbf{P}_{k \mid k}^{(j)}$ are the mean and covariance of the $\mathbf{j}:$ th component. In Appendix A, we state the Gaussian mixture filter equations used in our simulations.

\section{CPHD WITH SPAWNING}

As we saw in the previous section, the original CPHD filter is derived assuming a process model where new targets appear through spontaneous birth only, and not by birth or spawning as in the PHD filter. However, in some applications, it might be of interest to include a model for spawning also in the CPHD filter. In this section we discuss how the addition of a spawning model affects the CPHD equations (14)-(17), and we derive new equations for the steps that differ from the original filter. The derivations are based on the same assumptions and approximations as the original CPHD, except for the process model which here includes spawning of targets according to (2):

- New targets can appear through spontaneous birth or through spawning from existing targets. The intensity of targets from the birth process is denoted $b_{k}(\mathbf{x})$ and the spawning intensity for a single target with state $\mathbf{x}^{\prime}$ is given by $s_{k \mid k-1}\left(\mathbf{x} \mid \mathbf{x}^{\prime}\right)$. The corresponding cardinality distributions are denoted $p_{\mathrm{b}}(n)$ and $p_{\mathrm{s}}(n \mid \mathbf{x})$, respectively.

That is, the only change compared to the original CPHD setting is a new process model. The update in (6) is preformed by a multiplication of the predicted multi-target density $p\left(X_{k} \mid Z_{1: k-1}\right)$ by a likelihood $p\left(Z_{k} \mid X_{k}\right)$. Hence, given the predicted density, the update step does not take the process model into consideration, and (16)-(17) will not be affected when 
introducing a spawning model. The prediction formula, (5), on the other hand, is clearly dependent on the process model $p\left(X_{k} \mid X_{k-1}\right)$ and the original CPHD prediction, described by (14) and (15), will no longer be valid. In the remainder of this section we discuss and derive new prediction equations including target spawning.

\section{A. Prediction of the intensity function}

We start by discussing the prediction step for the intensity function. Since the process model and the assumptions in the PHD filter and our CPHD filter with spawning are very similar, a natural starting point is to study the original derivation of the intensity prediction in PHD [9].

The derivations in [9] are based on the process model (2) and on the assumptions that the motion of a target is independent of all other targets and that survival, birth and spawning of targets are independent of each other. Since the model and the assumption are identical to those used in the CPHD filter with spawning, we can adopt the intensity prediction equation from PHD (12) for our filter. Consequently, the intensity prediction for the CPHD filter with spawning is

$$
\begin{aligned}
v_{k \mid k-1}(\mathbf{x}) & =b_{k}(\mathbf{x})+\int\left\{P_{\mathrm{s}}\left(\mathbf{x}^{\prime}\right) p_{k \mid k-1}\left(\mathbf{x} \mid \mathbf{x}^{\prime}\right)\right. \\
& \left.+\int s_{k \mid k-1}\left(\mathbf{x} \mid \mathbf{x}^{\prime}\right)\right\} v_{k-1 \mid k-1}\left(\mathbf{x}^{\prime}\right) d \mathbf{x}^{\prime}
\end{aligned}
$$

This equation describes the predicted intensity of targets as the sum of the birth intensity and the intensities of surviving and spawning targets given the intensity $v_{k-1 \mid k-1}(\mathbf{x})$. For details on the derivation of this equation and a summary of the background theory, we refer to [9].

Note that, in reality, the predicted RFS is not an i.i.d. cluster process as assumed in the CPHD filter derivation. This is because the spawned targets are not independent of the targets from which they originate ${ }^{1}$, and therefore we, as in the PHD filter with spawning, approximate the predicted RFS as i.i.d.

\section{B. Prediction of the cardinality function}

In this section we derive the cardinality prediction equation for the CPHD filter with spawning. The derivations follow the approach in [25], that is, we use traditional statistics instead of finite set statistics, which is used in the original derivations. The reason for using traditional statistics is that the derivations become more straightforward and understandable also for readers unfamiliar with the concepts of FISST. Moreover, it provides a formula that allows us to derive explicit expressions for the spawning models given in Section V. For those more familiar with FISST, an overview of an alternative derivation can be found in [26].

The objective is to find an expression for the predicted cardinality, $p_{k \mid k-1}(n)=\operatorname{Pr}\left\{n_{k}=n \mid Z_{1: k-1}\right\}$, when the process model includes both birth and target spawning. We start by marginalizing over the number of targets at time $k-1$,

\footnotetext{
${ }^{1}$ This is also true for the posterior in the CPHD filter though the prior in the next recursion is again assumed to be (approximated as) an i.i.d. cluster
}

$$
\begin{aligned}
& \operatorname{Pr}\left\{n_{k} \mid Z_{1: k-1}\right\}=\sum_{n_{k-1}=0}^{\infty} \operatorname{Pr}\left\{n_{k}, n_{k-1} \mid Z_{1: k-1}\right\} \\
& =\sum_{n_{k-1}=0}^{\infty} \operatorname{Pr}\left\{n_{k} \mid n_{k-1}, Z_{1: k-1}\right\} \operatorname{Pr}\left\{n_{k-1} \mid Z_{1: k-1}\right\} .
\end{aligned}
$$

We recognize the factor $\operatorname{Pr}\left\{n_{k-1} \mid Z_{1: k-1}\right\}$ as the known cardinality distribution from the previous time instance, $p_{k-1 \mid k-1}(n)$. The first factor is found by introducing $i_{k}$ as the number of targets that survive from time $k-1$ to $k$, and marginalize over this variable,

$$
\begin{aligned}
& \operatorname{Pr}\left\{n_{k} \mid n_{k-1}, Z_{1: k-1}\right\}=\sum_{i_{k}=0}^{\min \left\{n_{k-1}, n_{k}\right\}} \operatorname{Pr}\left\{n_{k}, i_{k} \mid n_{k-1}\right\} \\
& =\sum_{i_{k}=0}^{\min \left\{n_{k-1}, n_{k}\right\}} \operatorname{Pr}\left\{n_{k} \mid i_{k}, n_{k-1}\right\} \operatorname{Pr}\left\{i_{k} \mid n_{k-1}\right\} .
\end{aligned}
$$

The probability $\operatorname{Pr}\left\{i_{k} \mid n_{k-1}\right\}$, accounting for the surviving targets, is identical to the corresponding factor in (15), and is found using the state dependent probability of survival and the assumption that existing targets continue to exist or disappear independently of each other,

$$
\begin{aligned}
& \operatorname{Pr}\left\{i_{k} \mid n_{k-1}\right\} \\
& =\left(\begin{array}{c}
n_{k-1} \\
i_{k}
\end{array}\right) \frac{\left\langle P_{\mathrm{s}}, v_{k-1 \mid k-1}\right\rangle^{i_{k}}\left\langle\left(1-P_{\mathrm{s}}\right), v_{k-1 \mid k-1}\right\rangle^{n_{k-1}-i_{k}}}{\left\langle 1, v_{k-1 \mid k-1}\right\rangle^{n_{k-1}}} .
\end{aligned}
$$

The factor $\operatorname{Pr}\left\{n_{k} \mid i_{k}, n_{k-1}\right\}$ in (25) describes the probability that $n_{k}-i_{k}$ new targets appear at the scene. In the original version of the CPHD, appearing targets are modeled by a birth process only for which $\operatorname{Pr}\left\{n_{k} \mid i_{k}, n_{k-1}\right\}=p_{\mathrm{b}}\left(n_{k}-i_{k}\right)$. This given the complete expression for the cardinality prediction as

$$
\begin{aligned}
p_{k \mid k-1}(n) & =\sum_{n^{\prime}=0}^{\infty} p_{k-1 \mid k-1}\left(n^{\prime}\right) \\
& \times \sum_{i=0}^{\min \left\{n, n^{\prime}\right\}}\left(\begin{array}{c}
n^{\prime} \\
i_{k}
\end{array}\right)\left\langle P_{\mathrm{s}}, v_{k-1 \mid k-1}\right\rangle^{i} \\
& \times \frac{\left\langle\left(1-P_{\mathrm{s}}\right), v_{k-1 \mid k-1}\right\rangle^{n_{k-1}-i}}{\left\langle 1, v_{k-1 \mid k-1}\right\rangle^{n^{\prime}}} p_{\mathrm{b}}(n-i),
\end{aligned}
$$

which is the same expression as in (15) but with a different order of summation.

Here, on the other hand, new targets can appear through either spawning or spontaneous birth, and by introducing $s_{k}$ as the total number of spawned targets at time $k$ we get

$$
\begin{aligned}
\operatorname{Pr}\left\{n_{k} \mid i_{k}, n_{k-1}\right\} & =\sum_{s_{k}=0}^{n_{k}-i_{k}} \operatorname{Pr}\left\{n_{k}, s_{k} \mid i_{k}, n_{k-1}\right\} \\
& =\sum_{s_{k}=0}^{n_{k}-i_{k}} \operatorname{Pr}\left\{n_{k} \mid s_{k}, i_{k}, n_{k-1}\right\} \operatorname{Pr}\left\{s_{k} \mid i_{k}, n_{k-1}\right\} \\
& =\sum_{s_{k}=0}^{n_{k}-i_{k}} p_{\mathrm{b}}\left(n_{k}-i_{k}-s_{k}\right) \operatorname{Pr}\left\{s_{k} \mid n_{k-1}\right\} .
\end{aligned}
$$


TABLE I: The spawning events in the example.

\begin{tabular}{|c|c|c|}
\hline Target 1 & Target 2 & Target 3 \\
\hline 3 & 0 & 0 \\
2 & 1 & 0 \\
2 & 0 & 1 \\
1 & 2 & 0 \\
1 & 1 & 1 \\
1 & 0 & 2 \\
0 & 3 & 0 \\
0 & 2 & 1 \\
0 & 1 & 2 \\
0 & 0 & 3 \\
\hline
\end{tabular}

The complete expression for the cardinality prediction with spawning becomes

$$
\begin{aligned}
p_{k \mid k-1}(n) & =\sum_{n^{\prime}=0}^{\infty} p_{k-1 \mid k-1}\left(n^{\prime}\right) \\
& \times \sum_{i=0}^{\min \left\{n, n^{\prime}\right\}}\left(\begin{array}{c}
n^{\prime} \\
i
\end{array}\right)\left\langle P_{\mathrm{s}}, v_{k-1 \mid k-1}\right\rangle^{i} \\
& \times \frac{\left\langle\left(1-P_{\mathrm{s}}\right), v_{k-1 \mid k-1}\right\rangle^{n^{\prime}-i}}{\left\langle 1, v_{k-1 \mid k-1}\right\rangle^{n^{\prime}}} \\
& \times \sum_{s=0}^{n-i} \operatorname{Pr}\left\{s_{k}=s \mid n_{k-1}=n^{\prime}\right\} p_{\mathrm{b}}(n-i-s) .
\end{aligned}
$$

The prediction formula in (29) might seem easy to handle, but depending on the choice of spawning model, $\operatorname{Pr}\left\{s_{k} \mid n_{k-1}\right\}$ may or may not be simple to compute. To better understand this probability, we study an example before presenting the general expression for $\operatorname{Pr}\left\{s_{k} \mid n_{k-1}\right\}$.

Example: Suppose that we are interested in finding the probability that three existing targets together spawn three targets at time $k$, i.e., we want to compute $\operatorname{Pr}\left\{s_{k}=3 \mid n_{k-1}=3\right\}$. To do that we need to consider all possible spawning events resulting in three new targets, see Table I where these are shown. Due to the assumption that all targets are identically distributed, the three targets are equally likely to spawn new targets and the single target spawning probability can be found as

$$
\begin{aligned}
\operatorname{Pr}\left\{s_{k} \mid n_{k-1}=1\right\} & =\int p_{\mathrm{s}}\left(s_{k} \mid \mathbf{x}_{k-1}\right) \\
\cdot & p\left(\mathbf{x}_{k-1} \mid Z_{1: k-1}, n_{k-1}=1\right) d \mathbf{x}_{k-1} .
\end{aligned}
$$

As a result, Table I can be summarized as the events:

1) One target spawns 3 targets while the other two do not spawn any targets. This can happen through $\left(\begin{array}{l}3 \\ 1\end{array}\right)=3$ equally likely spawning events.

2) One target spawns 2 targets, another spawns 1 , and the last target does not spawn any targets. This can happen through $\left(\begin{array}{l}3 \\ 1\end{array}\right)\left(\begin{array}{l}2 \\ 1\end{array}\right)=6$ equally likely events.

3) All three targets spawn 1 target. This can only happen in $\left(\begin{array}{l}3 \\ 3\end{array}\right)=1$ way.

Since the existing targets are i.i.d. and spawn targets independently of each other, the total probability accounting for these events can be found as

$$
\begin{aligned}
\operatorname{Pr}\left\{s_{k}=3 \mid n_{k-1}=3\right\}= & 3 \cdot \operatorname{Pr}\{3 \mid 1\} \operatorname{Pr}\{0 \mid 1\}^{2} \\
& +6 \cdot \operatorname{Pr}\{2 \mid 1\} \operatorname{Pr}\{1 \mid 1\} \operatorname{Pr}\{0 \mid 1\} \\
& +\operatorname{Pr}\{1 \mid 1\}^{3},
\end{aligned}
$$

where $\operatorname{Pr}\{a \mid b\}$ is shorthand for the spawning probability $\operatorname{Pr}\left\{s_{k}=a \mid n_{k-1}=b\right\}$.

$\square$ Following the idea from the example, the general probability $\operatorname{Pr}\left\{s_{k}=q \mid n_{k-1}\right\}$ can be found by considering in how many ways $n_{k-1}$ targets can spawn $s_{k}=q$ new targets. Denoting the number of targets that spawns $i$ new targets by $m_{i}$, we need to consider all $\left(m_{0}, m_{1}, \ldots, m_{q}\right)$ in the set $\mathcal{M}=\left\{\left(m_{0}, m_{1}, \ldots, m_{q}\right): m_{i} \geq\right.$ $\left.0,0 \cdot m_{0}+1 \cdot m_{1}+\ldots q \cdot m_{q}=q, \sum_{i=0}^{q} m_{i}=n_{k-1}\right\}$. Then the sought probability can be written in the general form

$$
\begin{aligned}
\operatorname{Pr}\left\{s_{k}=q \mid n_{k-1}\right\} & =\sum_{\mathcal{M}}\left(\begin{array}{c}
n_{k-1} \\
m_{0}
\end{array}\right)\left(\operatorname{Pr}\left\{s_{k}=0 \mid n_{k-1}=1\right\}\right)^{m_{0}} \\
& \times\left(\begin{array}{c}
n_{k-1}-m_{0} \\
m_{1}
\end{array}\right)\left(\operatorname{Pr}\left\{s_{k}=1 \mid n_{k-1}=1\right\}\right)^{m_{1}} \\
& \cdots\left(\begin{array}{c}
n_{k-1}-m_{1}-\ldots-m_{q-1} \\
m_{q}
\end{array}\right) \\
& \times\left(\operatorname{Pr}\left\{s_{k}=q \mid n_{k-1}=1\right\}\right)^{m_{q}} .
\end{aligned}
$$

Due to the combinatorial nature of the sum in (32), the spawning probability might be impractical to calculate in its general form. However, we will show that for specific spawning models it is possible to derive either exact or approximate expressions that are computationally tractable to work with. Furthermore, given such an expression, and if the probability of survival and the birth and spawning parameters are independent of time and target state, the cardinality prediction in (29) can be performed as

$$
p_{k \mid k-1}(n)=\sum_{n^{\prime}=0}^{\infty} M\left(n, n^{\prime}\right) p_{k-1 \mid k-1}\left(n^{\prime}\right),
$$

where the so called transition matrix $\mathrm{M}$, given by

$$
\begin{aligned}
& M\left(n, n^{\prime}\right)=\operatorname{Pr}\left\{n_{k}=n \mid n_{k-1}=n^{\prime}\right\} \\
& =\sum_{i=0}^{\min \left(n, n^{\prime}\right)}\left(\begin{array}{c}
n^{\prime} \\
i
\end{array}\right) P_{\mathrm{s}}^{i}\left(1-P_{\mathrm{s}}\right)^{n^{\prime}-i} \\
& \times \sum_{s=0}^{n-i} \operatorname{Pr}\left\{s_{k}=s \mid n_{k-1}=n^{\prime}\right\} p_{\mathrm{b}}\left(n-i-s_{k}\right),
\end{aligned}
$$

only needs to be computed once.

Examples on how to find $\operatorname{Pr}\left\{s_{k} \mid n_{k-1}\right\}$ for Bernoulli and Poisson spawning models is discussed in the following section, together with expressions for the cardinality prediction equation for some examples on birth and spawning models.

\section{SPECIFIC BIRTH AND SPAWNING MODELS}

There are several possible choices when it comes to modeling the birth and the spawning processes. In this section we consider two common cases, namely when the birth and the spawning models are either Bernoulli or Poisson, and 
we primarily focus on the spawning probabilities for these two models. First we discuss and state the expression for the spawning probability $\operatorname{Pr}\left\{s_{k} \mid n_{k-1}\right\}$ in (32), when $p_{\mathrm{s}}(n \mid \mathbf{x})$ is a Bernoulli distribution, and then we derive an approximate expression for the case when $p_{s}(n \mid \mathbf{x})$ is Poisson. Finally, we see how the different choices of spawning and birth models affect the expression for the predicted cardinality in (29).

\section{A. Bernoulli spawning model}

Assume that an existing target can spawn either zero or one target at each time instance. Then the cardinality of the spawning RFS is a Bernoulli distribution,

$$
p_{\mathrm{s}}(n \mid \mathbf{x})= \begin{cases}1-P_{\text {spawn }}(\mathbf{x}) & \text { if } \mathrm{n}=0 \\ P_{\text {spawn }}(\mathbf{x}) & \text { if } \mathrm{n}=1 \\ 0 & \text { if } n>1\end{cases}
$$

where the parameter $P_{\text {spawn }}(\mathbf{x})$ may be both time varying and state dependent. By studying the sum in (32) and recalling that an existing target can spawn at most one new target, we realize that $m_{i}=0$ for $i>1$ when using this spawning model. Consequently, the only possible non-zero term in (32) is if $s_{k}$ and $n_{k-1}$ are such that there exist $m_{0}=n_{k-1}-s_{k} \geq 0$ and $m_{1}=s_{k}$. The spawning probability is then given by

$$
\operatorname{Pr}\left\{s_{k} \mid n_{k-1}\right\}= \begin{cases}\left(\begin{array}{c}
n_{k-1} \\
s_{k}
\end{array}\right)\left(\bar{P}_{\text {spawn }}\right)^{s_{k}} & \\
\times\left(1-\bar{P}_{\text {spawn }}\right)^{n_{k-1}-s_{k}} & \text { if } s_{k} \leq n_{k-1} \\
0 & \text { if } s_{k}>n_{k-1}\end{cases}
$$

where

$$
\begin{aligned}
& \bar{P}_{\text {spawn }}=\operatorname{Pr}\left\{s_{k}=1 \mid n_{k-1}=1\right\} \\
& =\int P_{\text {spawn }}\left(\mathbf{x}_{k-1}\right) p\left(\mathbf{x}_{k-1} \mid Z_{1: k-1}, n_{k-1}=1\right) d \mathbf{x}_{k-1}
\end{aligned}
$$

is the probability that a single target spawns one new target.

\section{B. Poisson spawning model}

Now, assume that the number of spawned targets from an existing target is given by a Poisson distribution, i.e., the spawning cardinality is given as $p_{\mathrm{s}}(n \mid \mathbf{x})=\operatorname{Poiss}(n ; \lambda(\mathbf{x}))$. Then the probability of having $s_{k}$ spawned targets, conditioned on one existing target at time $k-1$, is

$$
\begin{aligned}
& \operatorname{Pr}\left\{s_{k} \mid n_{k-1}=1\right\} \\
& =\int p_{\mathrm{s}}\left(s_{k} \mid \mathbf{x}_{k-1}\right) p\left(\mathbf{x}_{k-1} \mid Z_{1: k-1}, n_{k-1}=1\right) d \mathbf{x}_{k-1} \\
& =\int \frac{e^{-\lambda\left(\mathbf{x}_{k-1}\right)} \lambda\left(\mathbf{x}_{k-1}\right)^{s_{k}}}{s_{k} !} p\left(\mathbf{x}_{k-1} \mid Z_{1: k-1}, n_{k-1}=1\right) d \mathbf{x}_{k-1}
\end{aligned}
$$

which can be seen as a weighted sum of Poisson distributions. In order to avoid evaluating the integral in (38) for all possible $s_{k}$ and then compute $\operatorname{Pr}\left\{s_{k} \mid n_{k-1}\right\}$ according to (32), we introduce an approximation that allows us to find a simpler expression for the spawning probability. We assume that the weighted sum in (38) can be approximated by a single Poisson distribution according to

$$
\operatorname{Pr}\left\{s_{k} \mid n_{k-1}=1\right\} \approx \frac{e^{-\bar{\lambda}} \bar{\lambda}^{s_{k}}}{s_{k} !},
$$

where $\bar{\lambda}$ is the expected number of spawned targets from a target whose state is described by the single target density $p\left(\mathbf{x}_{k-1} \mid Z_{1: k-1}\right)$. That is, $\bar{\lambda}$ can be found as

$$
\bar{\lambda}=\int \lambda\left(\mathbf{x}_{k-1}\right) p\left(\mathbf{x}_{k-1} \mid Z_{1: k-1}, n_{k-1}=1\right) d \mathbf{x}_{k-1} .
$$

Simulations has proved this approximation accurate for $\lambda(\mathbf{x})<1$ but, depending on the application, it can be satisfactory even for larger $\lambda$.

Using this approximation, the expression for the general probability $\operatorname{Pr}\left\{s_{k} \mid n_{k-1}\right\}$ can easily be found by convolution. The first step, i.e. going from an expression for $n_{k-1}=1$ to $n_{k-1}=2$, is:

$$
\begin{aligned}
\operatorname{Pr}\left\{s_{k} \mid n_{k-1}=2\right\} & =\operatorname{Pr}\left\{s_{k} \mid n_{k-1}=1\right\} * \operatorname{Pr}\left\{s_{k} \mid n_{k-1}=1\right\} \\
& \approx \sum_{j=0}^{s_{k}} \frac{e^{-\bar{\lambda}} \bar{\lambda}^{s_{k}-j}}{\left(s_{k}-j\right) !} \frac{e^{-\bar{\lambda}} \bar{\lambda}^{j}}{j !} \\
& =e^{-2 \bar{\lambda}} \sum_{j=0}^{s_{k}} \frac{s_{k} !}{s_{k} !} \frac{\bar{\lambda}^{s_{k}-j}}{\left(s_{k}-j\right) !} \frac{\bar{\lambda}^{j}}{j !} \\
& =\frac{e^{-2 \bar{\lambda}} \sum_{k}}{s_{k} !} \sum_{j=0}\left(\begin{array}{c}
s_{k} \\
j
\end{array}\right) \bar{\lambda}^{s_{k}-j} \bar{\lambda}^{j} \\
& =\frac{e^{-2 \bar{\lambda}}(2 \bar{\lambda})^{s_{k}}}{s_{k} !} \\
& =\operatorname{Poiss}\left(s_{k} ; 2 \bar{\lambda}\right),
\end{aligned}
$$

using the binomial formula

$$
(a+b)^{n}=\sum_{i=0}^{n}\left(\begin{array}{l}
n \\
i
\end{array}\right) a^{i} b^{n-i} .
$$

Following this procedure we can derive an expression for the general spawning probability, which can be shown to be

$$
\operatorname{Pr}\left\{s_{k} \mid n_{k-1}\right\} \approx \frac{e^{-n_{k-1} \bar{\lambda}}\left(n_{k-1} \bar{\lambda}\right)^{s_{k}}}{s_{k} !}=\operatorname{Poiss}\left(s_{k} ; \bar{\lambda} \cdot n_{k-1}\right) .
$$

Compared to the general expression in (32), this approximate formula is much simpler to handle and provides a tractable computation of the spawning probability.

\section{The cardinality prediction equation for different birth and spawning models}

Given a birth and a spawning model, we can find an expression for the last sum in the cardinality prediction equation (29),

$$
\sum_{s=0}^{n-i} \operatorname{Pr}\left\{s_{k}=s \mid n_{k-1}=n^{\prime}\right\} p_{\mathrm{b}}(n-i-s) .
$$

This sum describes the probability that $n-i$ new targets appear through spawning and birth, conditional on $n_{k-1}=n^{\prime}$. Having an expression for (44), we can state the full cardinality prediction equation for the current choice of models.

Here we consider two types of birth models, Bernoulli and Poisson, for which the cardinality distributions of the birth 
RFSs are

$$
p_{\mathrm{b}}(n)= \begin{cases}1-P_{\text {birth }} & \text { if } \mathrm{n}=0 \\ P_{\text {birth }} & \text { if } \mathrm{n}=1 \\ 0 & \text { if } n>1\end{cases}
$$

and

$$
p_{\mathrm{b}}(n)=\frac{e^{-\lambda_{\mathrm{b}}} \lambda_{\mathrm{b}}^{n}}{n !}
$$

respectively. The parameters $P_{\text {birth }}$ and $\lambda_{\mathrm{b}}$ are model parameters and can either be constant or time varying. Next, we present the resulting expressions for the sum in (44), given four different combinations of birth and spawning models.

Case 1: Poisson spawning and poisson birth: When both models are Poisson, the birth cardinality distribution is given by (46) and the spawning probability is found through the approximation in (43), $\operatorname{Pr}\left\{s_{k} \mid n_{k-1}\right\} \approx \operatorname{Poiss}\left(s_{k} ; \bar{\lambda} \cdot n_{k-1}\right)$. We can then find the desired sum as

$$
\begin{aligned}
& \sum_{s=0}^{n-i} \operatorname{Pr}\left\{s_{k}=s \mid n_{k-1}=n^{\prime}\right\} p_{\mathrm{b}}(n-i-s) \\
& \approx \sum_{s=0}^{n-i} \frac{e^{-\bar{\lambda} n^{\prime}}\left(\bar{\lambda} n^{\prime}\right)^{s}}{s !} \frac{e^{-\lambda_{\mathrm{b}}} \lambda_{\mathrm{b}}^{n-i-s}}{(n-i-s) !} \\
& =\frac{e^{-\left(\bar{\lambda} n^{\prime}+\lambda_{\mathrm{b}}\right)}\left(\bar{\lambda} n^{\prime}+\lambda_{\mathrm{b}}\right)^{n-i}}{(n-i) !}
\end{aligned}
$$

by using the same type of simplifications as in (41).

Case 2: Poisson spawning and Bernoulli birth: Considering this choice of models, the birth cardinality distribution is given by (45) while the spawning probability is found through the approximation in (43). Since the Bernoulli birth model describes the appearance of either zero or one born target, there will be a maximum of two non-zero terms in (44),

$$
\begin{aligned}
& \sum_{s=0}^{n-i} \operatorname{Pr}\left\{s_{k}=s \mid n_{k-1}=n^{\prime}\right\} p_{\mathrm{b}}(n-i-s) \\
& =\left\{\begin{array}{l}
p_{\mathrm{b}}(0) \operatorname{Pr}\left\{s_{k}=0 \mid n_{k-1}=n^{\prime}\right\} \\
p_{\mathrm{b}}(0) \operatorname{Pr}\left\{s_{k}=n-i \mid n_{k-1}=n^{\prime}\right\} \\
+p_{\mathrm{b}}(1) \operatorname{Pr}\left\{s_{k}=n-i-1 \mid n_{k-1}=n^{\prime}\right\} \quad \text { if } n-i \geq 0
\end{array}\right. \\
& \approx \begin{cases}\left(1-P_{\mathrm{birth}}\right) e^{-\bar{\lambda} n^{\prime}} & \text { if } n-i=0 \\
\left(1-P_{\mathrm{birth}}\right) \frac{e^{-\bar{\lambda} n^{\prime}}\left(\bar{\lambda} n^{\prime}\right)^{n-i}}{(n-i) !} & \text { if } n-i \geq 1 \\
+P_{\mathrm{birth}} \frac{e^{-\bar{\lambda} n^{\prime}\left(\bar{\lambda} n^{\prime}\right)^{n-i-1}}}{(n-i-1) !}\end{cases}
\end{aligned}
$$

Case 3: Bernoulli spawning and Bernoulli birth: When both the spawning model and the birth model are Bernoulli, described by (36) and (45), there are upper limits in both the number of spawned and born targets. There will be a maximum of one born target and $n_{k-1}=n^{\prime}$ spawned targets, since each of the $n_{k-1}$ existing target can spawn at most one target.
Hence,

$$
\begin{aligned}
& \sum_{s=0}^{n-i} \operatorname{Pr}\left\{s_{k}=s \mid n_{k-1}=n^{\prime}\right\} p_{b}(n-i-s) \\
& = \begin{cases}p_{\mathrm{b}}(1) \operatorname{Pr}\left\{s_{k}=n-i-1 \mid n_{k-1}=n^{\prime}\right\} \\
+p_{\mathrm{b}}(0) \operatorname{Pr}\left\{s_{k}=n-i \mid n_{k-1}=n^{\prime}\right\} & \text { if } n^{\prime} \geq n-i \\
p_{\mathrm{b}}(1) \operatorname{Pr}\left\{s_{k}=n-i-1 \mid n_{k-1}=n^{\prime}\right\} & \text { if } n^{\prime}=n-i-1 \\
0 & \text { if } n^{\prime}<n-i-1\end{cases} \\
& = \begin{cases}\left(1-P_{\text {birth }}\right)\left(\begin{array}{c}
n^{\prime} \\
n-i
\end{array}\right)\left(\bar{P}_{\text {spawn }}\right)^{n-i} & \text { if } n^{\prime} \geq n-i \\
\times\left(1-\bar{P}_{\text {spawn }}\right)^{n^{\prime}-(n-i)} & \text { if } n^{\prime}=n-i-1 \\
+P_{\text {birth }}\left(\begin{array}{c}
n^{\prime} \\
n-i-1
\end{array}\right)\left(\bar{P}_{\text {spawn }}\right)^{n-i-1} & \text { if } n^{\prime}<n-i-1 \\
\times\left(1-\bar{P}_{\text {spawn }}\right)^{n^{\prime}-(n-i-1)} & \\
P_{\text {birth }}\left(\bar{P}_{\text {spawn }}\right)^{n^{\prime}} & \\
0 & \end{cases}
\end{aligned}
$$

Case 4: Bernoulli spawning and Poisson birth: In this case the birth cardinality is given by (46). Since the spawning is Bernoulli, according to (36), each of the $n_{k-1}$ existing targets can spawn zero or one target and consequently there can not be more than $n_{k-1}=n^{\prime}$ spawned targets. Hence,

$$
\begin{aligned}
& \sum_{s=0}^{n-i} \operatorname{Pr}\left\{s_{k}=s \mid n_{k-1}=n^{\prime}\right\} p_{\mathrm{b}}(n-i-s) \\
& =\sum_{s=0}^{\min \left\{n-i, n^{\prime}\right\}} \operatorname{Pr}\left\{s_{k}=s \mid n_{k-1}=n^{\prime}\right\} p_{\mathrm{b}}(n-i-s) \\
& =\sum_{s=0}^{\min \left\{n-i, n^{\prime}\right\}}\left(\begin{array}{c}
n^{\prime} \\
s
\end{array}\right) \bar{P}_{\text {spawn }}^{s}\left(1-\bar{P}_{\text {spawn }}\right)^{n^{\prime}-s} \frac{e^{-\lambda_{\mathrm{b}}} \lambda_{\mathrm{b}}^{n-i-s}}{(n-i-s) !} .
\end{aligned}
$$

\section{Simulation Results}

In this section we evaluate the proposed algorithm for the scenario in Figure 1. To assess the performance of our filter, we use the average OSPA [22], accounting for accuracy in both cardinality and state estimates. Additionally, we consider the cardinality estimates separately by studying the RMSE and the average estimates including the standard deviation. The performance measures are computed using 200 Monte Carlo runs on the same underlying trajectory (but with randomly generated target and clutter measurements). The tracking result of the filter is compared to that of the original CPHD filter where no explicit spawning model is used.

In the simulations, both filters are implemented using a Gaussian Mixture filter; the original CPHD according to [14], [27], and the proposed filter using the equations in Appendix A. Further, merging and pruning is performed using the algorithm in [28], with a limitation of $J_{\max }=100$ Gaussians. The pruning threshold is set to $T_{\text {prune }}=0.001$ and the merging distance to $U=2$. The cardinality distribution is calculated for $n$ up to $N_{\max }=70$ and the number of targets at time $k$ is found as the maximum a posteriori (MAP) estimate,

$$
\hat{N}_{k \mid k}=\underset{n}{\operatorname{argmax}} p_{k \mid k}(n) .
$$


The filters are initialized with the prior cardinality $p_{0}(0)=0.4, p(1)=0.6, p_{0}(n)=0$ for $n>1$, and an intensity function consisting of one single Gaussian described by $w_{0}=0.6, m_{0}=[2,80,0,0]^{T}$ and $P_{0}=(\operatorname{diag}[10,10,5,5])^{2}$.

\section{A. Model assumptions}

The single target state $\mathrm{x}_{k}^{i}$ contains information about position and velocity in $\mathrm{x}$ - and $\mathrm{y}$-direction,

$$
\mathbf{x}_{k}^{i}=\left[x_{k}^{i}, y_{k}^{i}, \dot{x}_{k}^{i}, \dot{y}_{k}^{i}\right]^{T}
$$

and the probability that a target survives from one time instance until the next is independent of time and target state, such that $P_{\mathrm{s}, k}(\mathbf{x})=P_{\mathrm{s}}=0.97$. Each surviving target moves independently of all other targets according to the linear and Gaussian single target motion model

$$
\mathbf{x}_{k}=\mathbf{A} \mathbf{x}_{k-1}+\mathbf{v}_{k-1},
$$

where $\mathbf{v}_{k} \sim \mathcal{N}(\mathbf{0}, \mathbf{Q})$ is the process noise. The system matrix $\mathbf{A}$ and the noise covariance $\mathbf{Q}$ are

$$
\mathbf{A}=\left[\begin{array}{cc}
\mathbf{I}_{2 \times 2} & T_{\mathrm{s}} \mathbf{I}_{2 \times 2} \\
\mathbf{0}_{2 \times 2} & \mathbf{I}_{2 \times 2}
\end{array}\right], \quad \mathbf{Q}=\sigma_{Q}^{2}\left[\begin{array}{ll}
\frac{T_{\mathrm{s}}^{4}}{4} \mathbf{I}_{2 \times 2} & \frac{T_{\mathrm{s}}^{3}}{2} \mathbf{I}_{2 \times 2} \\
\frac{T_{\mathrm{s}}^{3}}{2} \mathbf{I}_{2 \times 2} & T_{\mathrm{s}}^{2} \mathbf{I}_{2 \times 2}
\end{array}\right]
$$

where $\mathbf{I}_{n \times n}$ and $\mathbf{0}_{n \times n}$ denote the $n \times n$ identity and zero matrices, $T_{\mathrm{s}}=0.2 \mathrm{~s}$ is the sampling time and $\sigma_{Q}=0.2$. In addition to the surviving targets at each time instance, there is also a possibility that new targets enter the observed scene. In our filter, the appearance of targets is modeled by a Bernoulli spawning process with constant probability $P_{\text {spawn }}$, and a Poisson birth process with constant parameter $\lambda_{\mathrm{b}}$. In the original CPHD filter we use a Poisson birth process, also with a constant parameter. The impact of the birth and the spawning parameters is further discussed in Section VI-B.

Each target is detected by the sensor with a time and state independent probability $P_{\mathrm{d}, k}(\mathbf{x})=P_{\mathrm{d}}=0.98$, and conditioned on detection, the target-generated measurement is described by the single-target measurement model,

$$
\mathbf{z}_{k}=\mathbf{H} \mathbf{x}_{k}+\mathbf{w}_{k} .
$$

Here, $\mathbf{w}_{k} \sim \mathcal{N}(\mathbf{0}, \mathbf{R})$ is the measurement noise and the matrices $\mathbf{H}$ and $\mathbf{R}$ are given as

$$
\mathbf{H}=\left[\begin{array}{ll}
\mathbf{I}_{2 \times 2} & \mathbf{0}_{2 \times 2}
\end{array}\right], \quad \mathbf{R}=\sigma_{R}^{2} \mathbf{I}_{2 \times 2}
$$

with $\sigma_{R}=0.5$. The matrix $\mathbf{H}$ in (56) implies that the received measurements are noisy observations of the 2-dimensional target position. In addition to the target-generated measurements, the received measurement set also contains clutter. The clutter RFS is a Poisson process with intensity $\kappa(\mathbf{z})=\lambda_{\mathrm{c}} V u(\mathbf{z})$, where $u(\mathbf{z})$ is a uniform distribution over the observed area $V=100 \times 100 \mathrm{~m}^{2}$, and where $\lambda_{\mathrm{c}}=3.5 \times 10^{-3}$ (giving an average of 35 clutter measurements per scan).

It is worth remembering that there is no requirement for the process and the measurement models to be linear. If any of the two models is nonlinear, the Kalman equations used in the prediction and the measurement update can be replaced by, for example, the corresponding Extended Kalman filter (EKF) or Unscented Kalman filter (UKF) equations as in [24].

\section{B. Results}

The scenario, depicted in Figure 1, starts with a single target and, through spawning, the number of targets grows to 8 before decreasing. As aforementioned, the spawning model in our

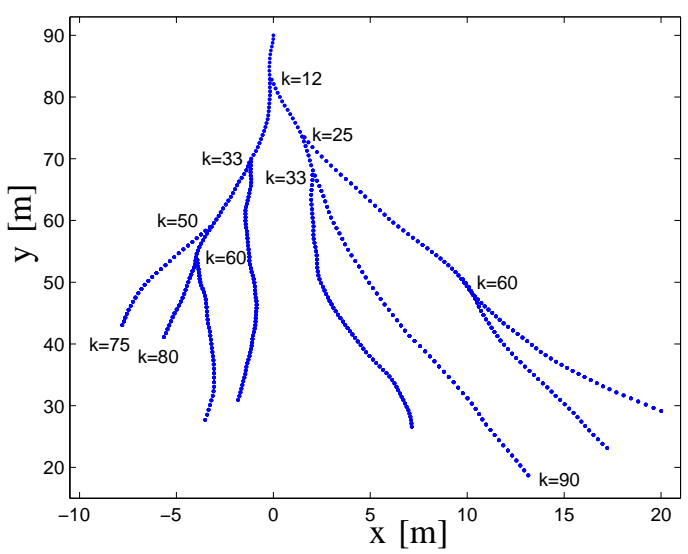

Figure 1: The target trajectories. The spawning events and the disappearance of targets are marked by the corresponding discrete time instance. For example, there is a spawning event at $k=12$ and at $k=60$ there are two spawned targets. Both spawning and survival is manually controlled.

filter is Bernoulli, while the birth model is Poisson, both with constant parameters. Naturally, as illustrated in Figure 2, the values of these parameters will affect the filter performance. The figure shows the mean OSPA and the mean standard deviation in the cardinality estimates for different choices of $P_{\text {spawn }}$ and $\lambda_{\mathrm{b}}$, given the models and parameters previously stated. In Figure 2(a), the mean OSPA has its lowest values for choices of parameters such that $P_{\text {spawn }} \in[0.04,0.1]$ while $\lambda_{\mathrm{b}} \in[0.01,0.04]$. It is important to remember that in the original CPHD filter the parameter set must be chosen such that $P_{\text {spawn }}=0$, i.e., from the leftmost column in the figure, while CPHD with spawning can use any combination of the two parameters. Considering this, we see that it is possible to achieve better performance, in mean OSPA sense, with our proposed filter than with the original CPHD filter. This gain in performance is however at the price of a higher standard deviation in the cardinality estimates, as shown in Figure 2(b). Based on these insights, in the subsequent simulations, we use $\lambda_{\mathrm{b}}=0.01$ and $P_{\text {spawn }}=0.06$ in the filter with a spawning model and $\lambda_{\mathrm{b}}=0.08$ in the original CPHD filter.

The illustrations in Figure 2 show the mean OSPA and the mean standard deviation, but it is also of interest to investigate how the filters behave over time during the scenario. We start by discussing the cardinality estimates over time and compare the behavior of the two filters.

The average cardinality estimates are shown in Figure 3 together with the standard deviation and the true number of targets. Here we see that the proposed method responds faster to an increase in target number, when it is due to spawning, than the original CPHD. Furthermore, our method is able to form unbiased estimates while the original CPHD algorithm clearly 


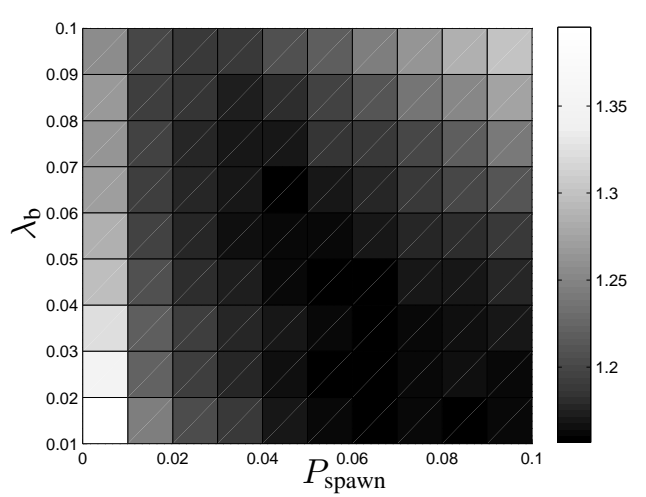

(a) The mean OSPA computed using $p=2$ and $c=5$.

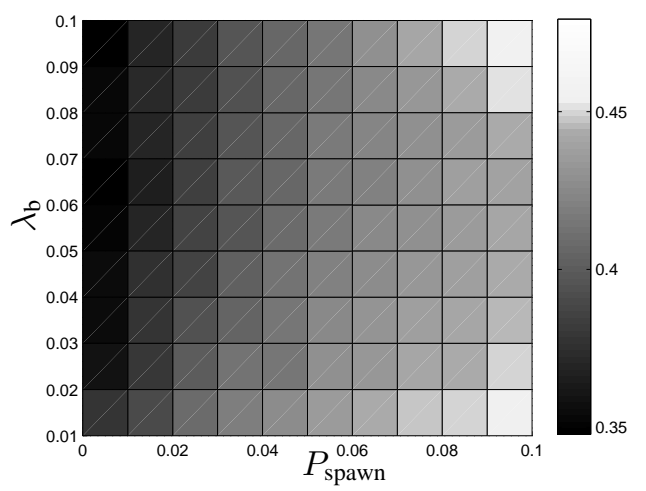

(b) The average standard deviation in the cardinality estimates.

Figure 2: The mean OSPA (a) and the mean standard deviation (b), for different values of $P_{\text {spawn }}$ and $\lambda_{\mathrm{b}}$. A darker color corresponds to a smaller mean OSPA, as indicated by the color bars. The values have been computed using 100 runs on the trajectory in Figure 1 , but with randomly generated measurements and clutter.

underestimates the number of targets. This, however, comes at the expense of somewhat increased estimation variance. The reason for the increase is due to the simple spawning model used in this example where probability mass is simply added proportional to the number of estimated targets. Studying Figure 2(b) also shows that this behavior is to be expected.

In Figure 4, we see the variation in the average cardinality error for the two methods. We see that the error curve for the original CPHD has clear peaks that coincide with the time instances where new targets appear by spawning, and that these peaks are significantly reduced by the addition of a spawning model. Thus, the CPHD filter with spawning detects a spawned target faster than the original filter. On the other hand, the cardinality error between the spawning events is slightly larger for the CPHD filter with spawning. Again, this is due to the increased variance in the cardinality estimates discussed above.

So far we have considered the cardinality estimation, but since we are also interested in the estimation of the individual targets states, we compute the average OSPA over time for the scenario. When computing OSPA we use the Euclidian distance together with the parameters $p=2$ and $c=5$,

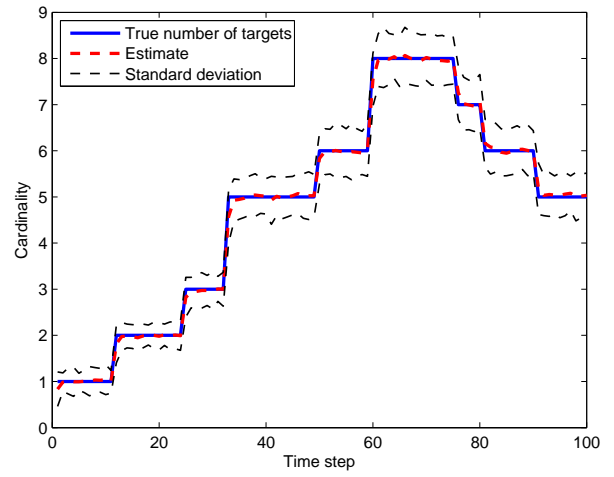

(a) CPHD with spawning

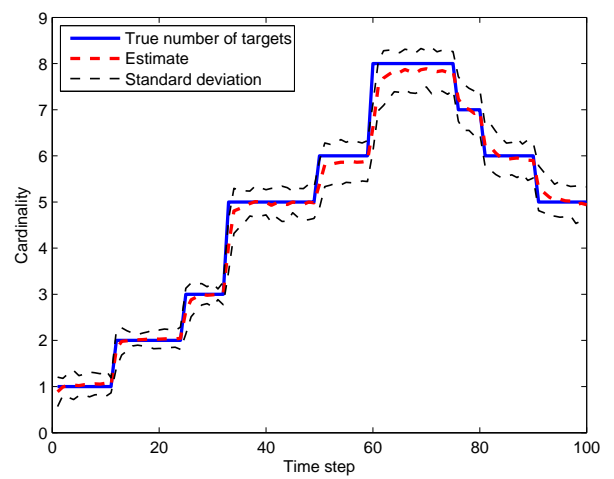

(b) Original CPHD

Figure 3: Cardinality estimates

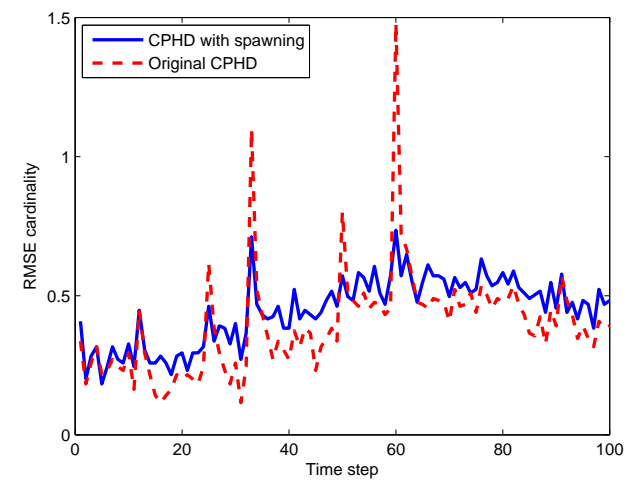

Figure 4: RMSE for the cardinality estimates

and the result is illustrated in Figure 5. Again we see clear peaks for the original CPHD at the time instances of the spawning events, and especially at the discrete time instances $k=33$ and $k=60$, where two targets are spawned at the same time. These peaks are, yet again, reduced when using the CPHD filter with spawning. To summarize the filter performance for this scenario, the incorporation of a spawning model apparently provides unbiased estimates of the number of targets that quickly detects changes in the cardinality at the moments of spawning. Although, we pay with a higher variance in the estimate, when considering both the cardinality and state estimates by computing the average OSPA, our 


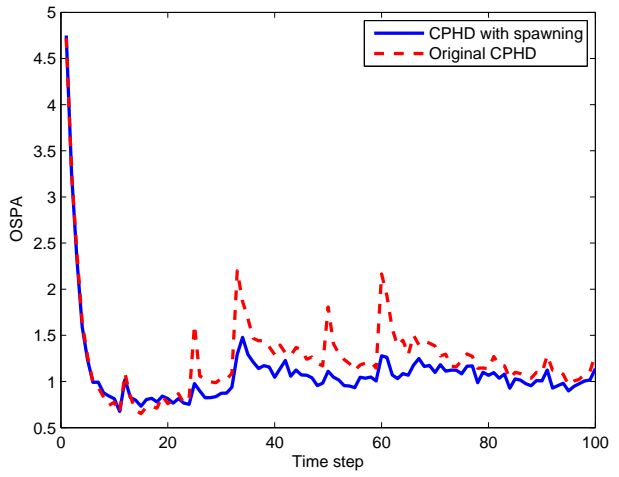

Figure 5: Average OSPA

proposed algorithm performs better during most parts of the scenario. Worth noting is that the spawning model used for generating the trajectories in figure 1 is manually controlled, i.e., deterministic. As such, there is a model mismatch between the simple spawning model used in the filter (constant spawning probability) and the simulation model (high spawning probability at discrete times). We expect that the use of a spawning model more adapted to the specific scenario would result in even better performance.

\section{CONCLUSIONS}

In this paper we have presented a generalized version of the CPHD filter incorporating an explicit spawning model. Compared to the original CPHD filter, the proposed filter is computationally more complex, and the increase in complexity depends on the choice of spawning model. One reason is that the derived equation for prediction of the cardinality distribution includes a model dependent probability which in its general form is impractical and computationally intractable. However, we have shown that for a Bernoulli model, the spawning probability is found through a simple and exact expression. Moreover, given a Poisson model, we have derived an approximate expression for the spawning probability resulting in a computationally efficient formula.

Evaluation on an illustrative scenario, using constant spawning parameter, shows promising results for the proposed algorithm. The evaluation shows that the filter responds faster to a change in target number due to spawning and that we gain performance, in OSPA sense, by adding the spawning model compared to the original CPHD filter. However, how the filter performs in real applications, dealing with more complex scenarios or using a more sophisticated spawning model, is hard to predict and would be interesting to investigate further.

\section{APPENDIX}

In the appendix we summarize the equations used for implementing the Gaussian mixture version of our proposed filter.

\section{A. Gaussian mixture CPHD with spawning}

The implementation of the Gaussian mixture version of the CPHD filter with spawning is based on [27] and [13]. The intensity function from time $k-1$ is described as a weighted sum of Gaussians

$v_{k-1 \mid k-1}(\mathbf{x})=\sum_{j=0}^{J_{k-1 \mid k-1}} w_{k-1 \mid k-1}^{(j)} \mathcal{N}\left(\mathbf{x} ; \mathbf{m}_{k-1 \mid k-1}^{(j)}, \mathbf{P}_{k-1 \mid k-1}^{(j)}\right)$

where $\mathbf{m}_{k-1 \mid k-1}^{(j)}$ and $\mathbf{P}_{k-1 \mid k-1}^{(j)}$ are the mean and covariance of the j:th component.

Prediction: The prediction of each existing component is given by

$$
\begin{aligned}
w_{k \mid k-1}^{(j)} & =P_{\mathrm{s}} w_{k-1 \mid k-1}^{(j)} \\
\mathbf{m}_{k \mid k-1}^{(j)} & =\mathbf{A m}_{k-1 \mid k-1}^{(j)} \\
\mathbf{P}_{k \mid k-1}^{(j)} & =\mathbf{A P}_{k-1 \mid k-1}^{(j)} \mathbf{A}^{T}+\mathbf{Q} .
\end{aligned}
$$

Additional components are introduced by the Poisson birth model and Bernoulli spawning model. In our simulations the birth intensity $b(\mathbf{x})$ and the spawning intensity $s(\mathbf{x})$ are given as

$$
\begin{aligned}
b(\mathbf{x}) & =\sum_{i=1}^{J_{b, k}} w_{\mathrm{b}, k}^{(i)} \mathcal{N}\left(\mathbf{x} ; \mathbf{m}_{\mathrm{b}, k}^{(i)}, \mathbf{P}_{\mathrm{b}, k}^{(i)}\right)=w_{\mathrm{b}} \mathcal{N}\left(\mathbf{x} ; \mathbf{m}_{\mathrm{b}}, \mathbf{P}_{\mathrm{b}}\right) \\
s(\mathbf{x}) & =\sum_{j=1}^{J_{k-1 \mid k-1}} \sum_{i=1}^{J_{\mathrm{s}, k}} w_{k-1 \mid k-1}^{(j)} w_{\mathrm{s}, k}^{(i)} \mathcal{N}\left(\mathbf{x} ; \mathbf{m}_{\mathrm{s}, k}^{(j, i)}, \mathbf{P}_{\mathrm{s}, k}^{(j, i)}\right) \\
& =\sum_{j=1}^{J_{k-1 \mid k-1}} w_{k-1 \mid k-1}^{(j)} w_{\mathrm{s}} \mathcal{N}\left(\mathbf{x} ; \mathbf{m}_{\mathrm{s}, k}^{(j)}, \mathbf{P}_{\mathrm{s}, k}^{(j)}\right)
\end{aligned}
$$

where $w_{\mathrm{b}}=\lambda_{\mathrm{b}}, \mathbf{m}_{\mathrm{b}}=[0,50,0,0]^{T}$, $\mathbf{P}_{\mathrm{b}}=(\operatorname{diag}[30,30,5,5])^{2}, \quad w_{\mathrm{s}}=P_{\text {spawn }}, \quad \mathbf{m}_{\mathrm{s}, k}^{(j)}=$ $\mathbf{A} \mathbf{m}_{k-1 \mid k-1}^{(j)}=\mathbf{m}_{k \mid k-1}^{(j)}, \mathbf{P}_{\mathrm{s}, k}^{(j)}=\mathbf{A} \mathbf{P}_{k-1 \mid k-1}^{(j)} \mathbf{A}^{T}+\mathbf{Q}+\mathbf{Q}_{s}=$ $\mathbf{P}_{k \mid k-1}^{(j)}+\mathbf{Q}_{s}$ and $\mathbf{Q}_{\mathrm{s}}=(\operatorname{diag}[0.5,0.5,0.5,0.5])^{2}$.

The cardinality prediction formula using the Bernoulli spawning and the Poisson birth models with constant parameters is given as

$$
p_{k \mid k-1}(n)=\sum_{n^{\prime}=0}^{\infty} M\left(n, n^{\prime}\right) p_{k-1 \mid k-1}\left(n^{\prime}\right),
$$

where the transition matrix is

$$
\begin{aligned}
& M\left(n, n^{\prime}\right)=\sum_{i=0}^{\min \left\{n, n^{\prime}\right\}}\left(\begin{array}{c}
n^{\prime} \\
i
\end{array}\right) P_{\mathrm{s}}^{i}\left(1-P_{\mathrm{s}}\right)^{n^{\prime}-i} \\
& \times \sum_{s=0}^{\min \left\{n-i, n^{\prime}\right\}}\left(\begin{array}{c}
n^{\prime} \\
s
\end{array}\right)\left(P_{\text {spawn }}\right)^{s}\left(1-P_{\text {spawn }}\right)^{n^{\prime}-s} \frac{e^{-\lambda_{b}} \lambda_{b}^{n-i-s}}{(n-i-s) !} .
\end{aligned}
$$

Update: The update equations in the CPHD filter with spawning is identical to those of the original filter, thus, the used equations are found in [27].

\section{REFERENCES}

[1] S. Blackman and R. Popoli, Design and analysis of modern tracking systems. Norwood, MA: Artech House, 1999.

[2] Y. Bar-Shalom, X. R. Li, and T. Kirubarajan, Estimation with applicarions to tracking and navigation. New York, NY: Wiley, 2001. 
[3] Y. Bar-Shalom and E. Tse, "Tracking in a cluttered environment with probabilistic data association," Automatica, vol. 11, no. 5, pp. 451 - 460, 1975. [Online]. Available: http://www.sciencedirect.com/science/article/pii/0005109875900217

[4] T. Fortmann, Y. Bar-Shalom, and M. Scheffe, "Sonar tracking of multiple targets using joint probabilistic data association," IEEE Journal of Oceanic Engineering, vol. 8, no. 3, pp. 173 - 184, July 1983.

[5] D. Reid, "An algorithm for tracking multiple targets," Automatic Control, IEEE Transactions on, vol. 24, no. 6, pp. 843-854, Dec 1979.

[6] S. Blackman, "Multiple hypothesis tracking for multiple target tracking," IEEE Aerospace and Electronic Systems Magazine, vol. 19, no. 1, pp. $5-18$, Jan. 2004.

[7] R. Mahler, Statistical multisource-multitarget information fusion. Artech House, Inc. Norwood, MA, USA, 2007.

[8] B. Vo and B. Vo, "A random finite set conjugate prior and application to multi-target tracking," in Intelligent Sensors, Sensor Networks and Information Processing (ISSNIP), 2011 Seventh International Conference on. IEEE, 2011, pp. 431-436.

[9] R. Mahler, "Multitarget Bayes filtering via first-order multitarget moments," IEEE Transactions on Aerospace and Electronic Systems, vol. 39, no. 4, pp. 1152-1178, 2003.

[10] O. Erdinc, P. Willett, and Y. Bar-Shalom, "Probability hypothesis density filter for multitarget multisensor tracking," in 8th International Conference on Information Fusion, 2005, July 2005.

[11] R. Mahler, "PHD Filters of Higher Order in Target Number," IEEE Transactions on Aerospace and Electronic Systems, vol. 43, no. 4, 2007.

[12] B.-N. Vo, S. Singh, and A. Doucet, "Sequential Monte Carlo methods for multitarget filtering with random finite sets," IEEE Transactions on Aerospace and Electronic Systems, vol. 41, no. 4, pp. 1224 - 1245, oct. 2005.

[13] B. Vo and W. Ma, "The Gaussian mixture probability hypothesis density filter," IEEE Transactions on Signal Processing, vol. 54, no. 11, p. 4091, 2006

[14] B.-T. Vo, B.-N. Vo, and A. Cantoni, "The Cardinalized Probability Hypothesis Density Filter for Linear Gaussian Multi-Target Models," in Information Sciences and Systems, 2006 40th Annual Conference on, March 2006, pp. $681-686$.

[15] S. Singh, B. Vo, A. Baddeley, and S. Zuyev, "Filters for spatial point processes," SIAM Journal on Control and Optimization, vol. 48, no. 4, pp. 2275-2295, 2009.

[16] F. Caron, P. Del Moral, A. Doucet, and M. Pace, "On the conditional distributions of spatial point processes," Advances in Applied Probability, vol. 43, no. 2, pp. 301-307, 2011

[17] R. Mahler, B.-T. Vo, and B.-N. Vo, "CPHD Filtering With Unknown Clutter Rate and Detection Profile," IEEE Transactions on Signal Processing, vol. 59, no. 8, pp. $3497-3513$, aug. 2011.

[18] R. Mahler, "PHD filters for nonstandard targets, I: Extended targets," in 12th International Conference on Information Fusion, 2009. FUSION '09., July 2009, pp. $915-921$.

[19] U. Orguner, C. Lundquist, and K. Granström, "Extended target tracking with a cardinalized probability hypothesis density filter," in Proceedings of the 14th International Conference on Information Fusion (FUSION), 2011, July 2011, pp. $1-8$.

[20] K. Granström, C. Lundquist, and U. Orguner, "A Gaussian mixture PHD filter for extended target tracking," pp. $1-8$, July 2010.

[21] A. Pasha, B. Vo, H. Tuan, and W.-K. Ma, "Closed Form PHD Filtering for Linear Jump Markov Models," in 9th International Conference on Information Fusion, 2006, July 2006, pp. 1 -8.

[22] D. Schuhmacher, B.-T. Vo, and B.-N. Vo, "A Consistent Metric for Performance Evaluation of Multi-Object Filters," IEEE Transactions on Signal Processing, vol. 56, no. 8, pp. 3447 -3457, Aug. 2008.

[23] M. Guerriero, L. Svensson, D. Svensson, and P. Willett, "Shooting two birds with two bullets: How to find Minimum Mean OSPA estimates," in 13th Conference on Information Fusion (FUSION), 2010, July 2010, pp. $1-8$.

[24] B.-T. Vo, B.-N. Vo, and A. Cantoni, "Analytical Implementations of the Cardinalized Probability Hypothesis Density Filter," IEEE Transactions on Signal Processing, vol. 55, no. 7, 2007.

[25] D. Svensson, "An Alternative Derivation of the Cardinalized Probability Hypothesis Density Filter," Department of Signal and Systems, Chalmers University of Technology, Tech. Rep., 2008.

[26] M. Lundgren, L. Svensson, and L. Hammarstrand, "A CPHD Filter for Tracking with Spawning Models - including a FISST based derivation," Department of Signal and Systems, Chalmers University of Technology, Tech. Rep., 2012.
[27] M. Ulmke, O. Erdinc, and P. Willett, "Gaussian mixture cardinalized PHD filter for ground moving target tracking," in 10th International Conference on Information Fusion, 2007, July 2007, pp. 1 -8.

[28] D. Clark, K. Panta, and B.-N. Vo, "The GM-PHD Filter Multiple Target Tracker," in 9th International Conference on Information Fusion, 2006, July 2006, pp. $1-8$.

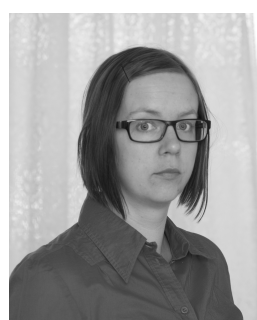

Malin Lundgren was born in Skövde, Sweden in 1979. She received the M.Sc degree in electrical engineering from Chalmers University of Technology, Gothenburg, Sweden, in 2004.

After a couple of years in the industry she returned to Chalmers where she is currently working as a $\mathrm{Ph} . \mathrm{D}$. student with interest in sensor fusion and extended object tracking, primarily for automotive safety applications.

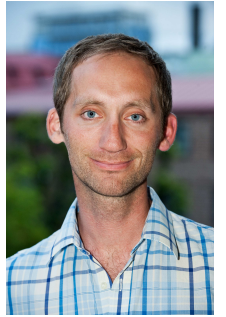

Lennart Svensson was born in Älvängen, Sweden in 1976. He received the M.S. degree in electrical engineering in 1999 and the Ph.D. degree in 2004, both from Chalmers University of Technology, Gothenburg, Sweden.

$\mathrm{He}$ is currently Associate Professor at the Signal Processing group, again at Chalmers University of Technology. His research interests include Bayesian inference in general, and nonlinear filtering and tracking in particular.

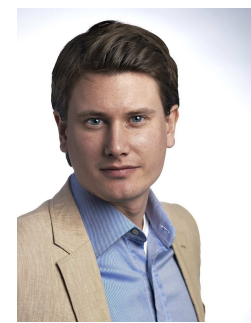

Lars Hammarstrand was born in Landvetter, Sweden in 1979. He received his M.Sc. and Ph.D. degree in electrical engineering from Chalmers University of Technology, Gothenburg, Sweden, in 2004 and 2010 , respectively.

From 2004 to 2011, he was with the Active Safety and Chassis Department at Volvo Car Corporation, Gothenburg, conducting research on tracking and sensor data fusion methods for active safety systems. Currently, Lars is a Postdoctoral Research Fellow at the Signal Processing group at Chalmers University of Technology where his main research interests are in the fields of estimation, sensor fusion and radar sensor modeling, especially with application to active safety systems. 